\title{
Reliability Analysis and Motion Simulation of Steel Coil Upender Clamp
}

\author{
Anyuan Jiao ${ }^{1, a}$ Fenghui Wang ${ }^{2, b}$ \\ ${ }^{1}$ The School of Higher Vocational Education, University of Science and Technology Liaoning, \\ Anshan, China \\ ${ }^{2}$ The School of Mechanical Engineering and Automation, University of Science and Technology \\ Liaoning, Anshan, China \\ E-mail: ${ }^{a}$ jjaay@163.com, ${ }^{b}$ V_kjc@163.com
}

Keywords: Reliability analysis, Motion simulation, SolidWorks, ADAMS

\begin{abstract}
A kind of new steel coil upender clamping device was designed. The model was set up by use of SolidWorks and the reliability analysis and motion simulation was further performed in ADAMS. Because of risk at steel coil upender, once the accident appears, it gently slowed-up production and damaged the device, and it could cause people's dying vitally, it is especially important to assure the longtime work and safety of device which we studied on. The results showed that the efficiency of turnover process of the device was high and the system could save driving force. These also provided some theory bases for the design of the device.
\end{abstract}

\section{Introduction}

Steel coil was laid aside horizontally after it was packed in sheet strip workplace. Then steel coil was transported to storehouse by horizontal type. But nation specified that coil transporting should be laid vertically because this can increase safety of laying and save covering space. In order to satisfy different customer's demand some steel coil factories established special coil turnover device, and have to provide two kinds of hangers to clamp horizontal and vertical coil. Therefore it is necessary for designing a new clamp to conveniently transporting and transferring between horizontal and vertical of steel coil [1]. This device combined the clamp body of a chain-type clamp and the turnover, which the principles of turnover mechanism are similar [2]. The new steel coil clamping device in this paper could carry out these functions which have introduced above.

\section{Structure and 3D Model}

The new upender clamp contains two main parts, clamping mechanism and upender device. The clamping mechanism consists of clamp end part, clamp body and fixed clamp finger, move clamp finger etc. Upender device consists of lifting lug device, hanging beam, rolling shaft, drive gear...etc. The solid model was built by using of SolidWorks software, which is a kind of 3D $\mathrm{CAD} / \mathrm{CAE}$ software and it appeared and was quickly expanded in recent years. It has well operation interface windows environment, and it is easy and convenient to build up 3D model [3]. The final assembly model of new upender clamping is shown in Fig.1. The clamping force doesn't depend on the rigidity of two legs to clamp works tightly, but depends on its dead weight after being hold up. This produced by a tilt angle between leg and slider makes steel coil more and more tightly. When electrical upender clamp works, at first to clamp the coil firmly, and then lift crane to raise the clamp body coils, up to a certain height to stop, then to turn the coil from the vertical into horizontal. The whole working process consists of two basic actions: the clamping action and upender action.

\section{Reliability Analysis of Coil Clamping}

Coil was clamped by the lifting device will bring a certain height, so the reliability of clamp is very important. After the coil was hold up, the main force is the friction generated by the pressure between the clamp legs and coils. This friction that was generated by the positive pressure on clamp 
legs is large enough to balance the coil's weight. Taking into account the actual work conditions, the oil or emulsion may infiltrate the clamp, and to ensure the safety and reliability, According to the theoretical analysis and Table 1, the maximum clamping diameter coil $(2 \mathrm{~m})$ when the friction coefficient of 0.1 is tentatively scheduled for to this design as the maximum lifting coil diameter, using a computer program, to meet the above-mentioned principles and compact to ensure the safety of the clamp body of the model analysis.

Table 1 The friction coefficient between steel and steel

\begin{tabular}{lllll}
\hline \multirow{2}{*}{$\begin{array}{l}\text { The friction coefficient } \\
\text { between steel and steel }\end{array}$} & \multicolumn{2}{l}{ Static friction coefficient } & \multicolumn{2}{l}{ Dynamic friction coefficient } \\
& $\begin{array}{l}\text { with } \\
\text { lubrication }\end{array}$ & $\begin{array}{l}\text { without } \\
\text { lubrication }\end{array}$ & $\begin{array}{l}\text { with } \\
\text { lubrication }\end{array}$ & $\begin{array}{l}\text { without } \\
\text { lubrication }\end{array}$ \\
\hline Smooth steel to steel & $0.1-0.12$ & 0.15 & $0.05-0.1$ & 0.1 \\
\hline
\end{tabular}

Many types of constraints can be used in ADAMS: Revolute, Translational, Cylinder, Spherical, Screw, Gear, Coupler, Fixed and so on. Add constraints should be selected constraint type, the role of parts, the role of point and direction. Add a constraint in the process, sometimes resulting redundant constraints, too many restrictions on their freedom of institutions, which often leads to interruption of the simulation analysis, this phenomenon can be controlled by the simulation model validation panel to check, then reduce the limit components bound by the same number of degrees of freedom corresponding redundant constraints can solve the problem. After all above has been done, add damping friction and motor in revolute joint and translational joint.

Before the simulation, should be the final test of the prototype model, out of the error implicit in the import process to ensure the accuracy of the simulation analysis and reliability. The use of model diagnostic tool to check inappropriate joints and constraints, is not binding parts, no quality of parts, prototypes of freedom and so on. Complete debug and test a model, you can clamp a virtual prototype simulation test. During the process of motion simulation, ADAMS/View automatically call ADAMS/Solver to complete three types of simulation: dynamic simulation analysis (Dynamic), by solving a series of nonlinear differential and algebraic equations, analysis greater than zero degrees of freedom of movement and complex systems in all forces; kinematic simulation (Kinematic), by solving a series of equations, degrees of freedom equal to zero to determine the motion analysis of motion of the system; static simulation (Static), by equilibrium conditions, solving component of the force of static analysis. According to clamp the actual work, the motion simulations were done on the prototype model. The materials of clamp are set to structural steel, there are two ways can be implemented simulation: (1) to clamp a uniform motion speed up the simulation; (2) to apply force to a volume approximately equal to the total weight of the upender clamp to carry out simulation. The first method used in this paper the simulation procedure of the clamp was divided into two steps: firstly, to simulate the clamp process; the second step, the lifting simulation were done while maintaining the clamp state.

In the first step to moving the slider body fixed to the moving clamp body and add a translational joint constrain between clamp body and static to the dynamic fixed joint between static clamp body and static clamp legs. The contact pressure was added between the static body of the slider and the coils, so that when the clamp will automatically generate a positive pressure on the coil from the clamp legs. The analysis results shown in Fig. 2, during in the first process, due to the clamp body sliding to coil, the slider body has not come into contact with the coil, so value of positive pressure in the first few seconds is 0 , when beginning to contact, the positive pressure began to rise. CONTACT_1 is pressure curve for the static body is between the slider and coil, CONTACT_2 is pressure curve for the moving body is between the slider and coil. The maximum positive pressure of CONTACT_1 up to $6 \times 10^{6} \mathrm{~N}$, positive pressure of CONTACT_ 2 can be achieved in $5 \times 10^{6} \mathrm{~N}$.

After positive pressure generated, the second step analysis will process. Clamp applied to the whole body a uniform up speed, check the coil relative to the sliding clamp leg acceleration. Respectively, to move clamp leg, static clamp was added between the legs and slide coil bound selected static friction coefficient of 0.1 , dynamic friction coefficient of 0.05 . Velocity on the given vector, the results were shown in Fig.3. The curve shows the initial stage of lifting, clamp legs and 
coil will have a little sliding, but to enter the steady state, the value of the coil acceleration is 0 , indicating the coil can be clamp firmly. Fig. 4 is positive pressure curve between a static body and coil, or between the slider body and the coil.

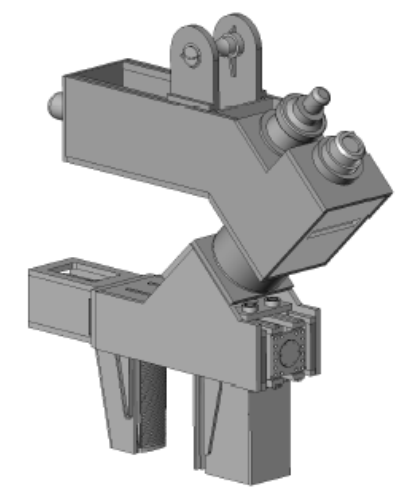

Fig. $13 \mathrm{D}$ model of upender clamp

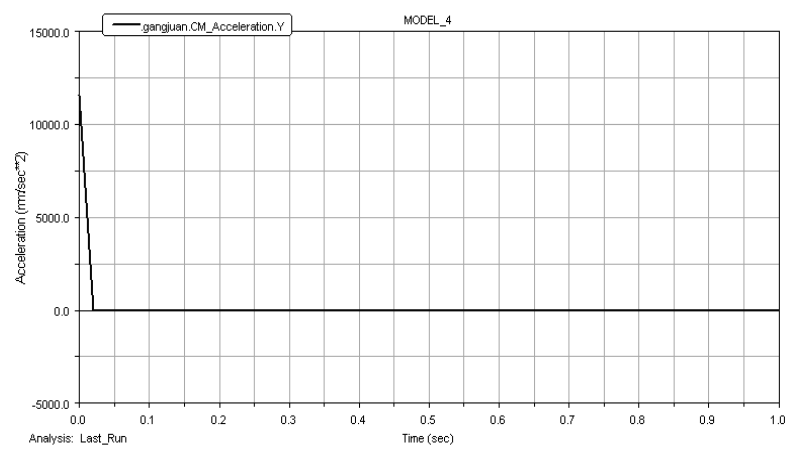

Fig. 3 Coil acceleration as friction coefficient of 0.1

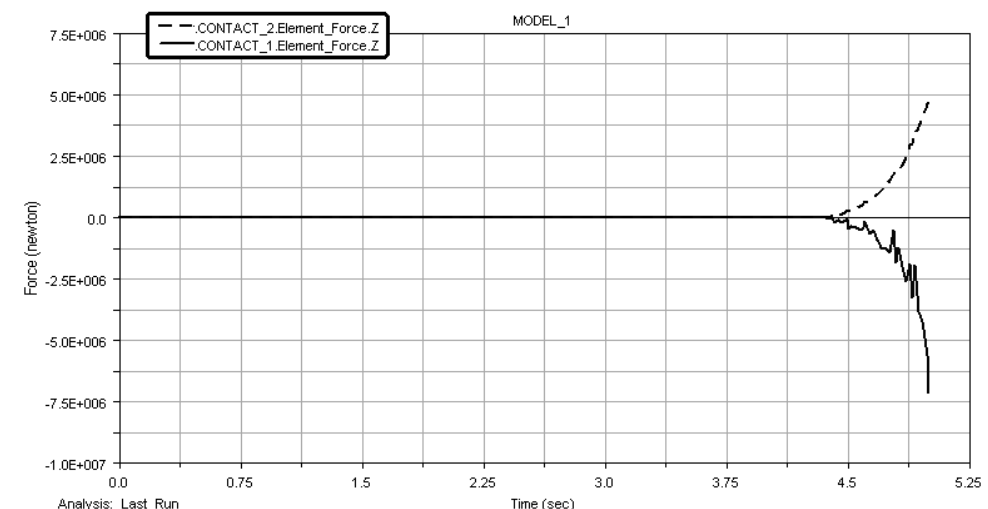

Fig. 2 Pressure curve of clamp legs

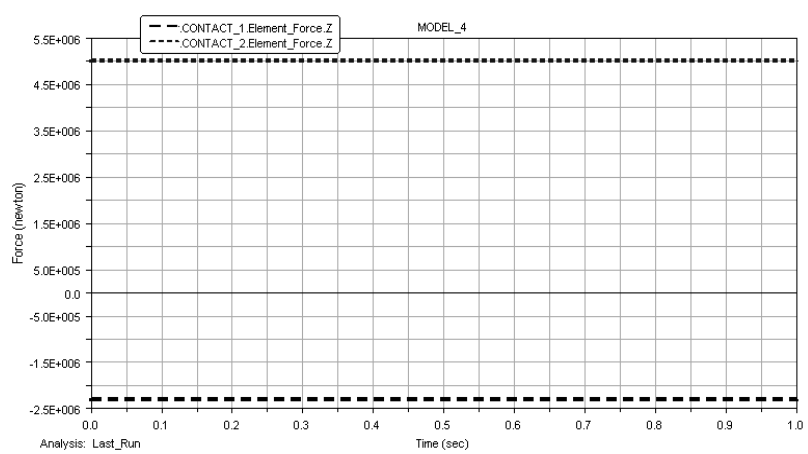

Fig. 4 Positive pressure curve

It can be seen from the figure, positive pressure between static body and the coil maintained at about $5 \times 10^{6} \mathrm{~N}$, and the positive pressure between the slider body and the coil will remain at $2.2 \times 10^{6} \mathrm{~N}$. The gravity of coils is $30 \mathrm{t}$, and the theoretical positive pressure was calculated in Eq. 1 .

$$
G=2 f \Rightarrow f=G / 2=147000(N) \Rightarrow P=f / f_{s}=147000 / 0.1=1.47 \times 10^{6}(N)
$$

Which, G- rated maximum weight of coil, $f$ - the side friction, P-the side pressure, $f_{\mathrm{s}^{-}}$static friction coefficient.

The analysis conclusion shows: $2.2 \times 10^{6}>1.47 \times 10^{6} \mathrm{~N}$, so the upender clamp can work tightly.

\section{Motion Analysis in ADAMS}

ADAMS software is mainly used for simulation analysis of large-displacement and complicated system. The simulation analyses based on the traditional machine system contain six basic steps: Identifying the large-displacement movement machine system; Simplify system; build up the calculator model of simplifying system; Building up system's motion equation; Solve the motion equation; Output simulation analysis results.

Invert Force. This dynamics simulation belongs to contrary dynamics analysis, where the velocity has been already known, then constraint force between components is to be solved. By Newton-Euler motion equation of machine system [4]. The model built with SolidWorks, some components such as lead screw, gear mechanism, electrical motor...etc was compressed, and then imported the model into ADAMS. Then the material attribute for each component are added respectively. According to the design request this handling device probably $6 \mathrm{t}$, the nominal weight of steel coil which could be clamp is $30 \mathrm{t}$. 
According to the request, the invert process needs to consume $10 \mathrm{~s}$; the angular velocity of rolling shaft is $0.3 \mathrm{rad} / \mathrm{s}$. Added revolve driving, whose angular velocity is $0.3 \mathrm{rad} / \mathrm{s}$ and step number is 50 , ADAMS software will build up motion equation automatically. According to initial conditions which added, forces between components can be solved. Fig. 5 and Fig. 6 are the curve of driving torque and driving force respectively, which analyzed by ADAMS $\backslash$ Postprocessor. From the curve that driving force is probably $520 \mathrm{~N}$ at the time of start rolling that driving torque is about $1.46 \times 10^{3} \mathrm{~N} \cdot \mathrm{m}$. The biggest value at $6.6 \mathrm{~s}$ is appeared, whose value is $1.52 \times 10^{3} \mathrm{~N} \cdot \mathrm{m}$. Therefore, according to driving force and driving torque by simulation analysis, we could estimate the gearing shaft's power. Then according to this power, suitable electrical motor can be selected.

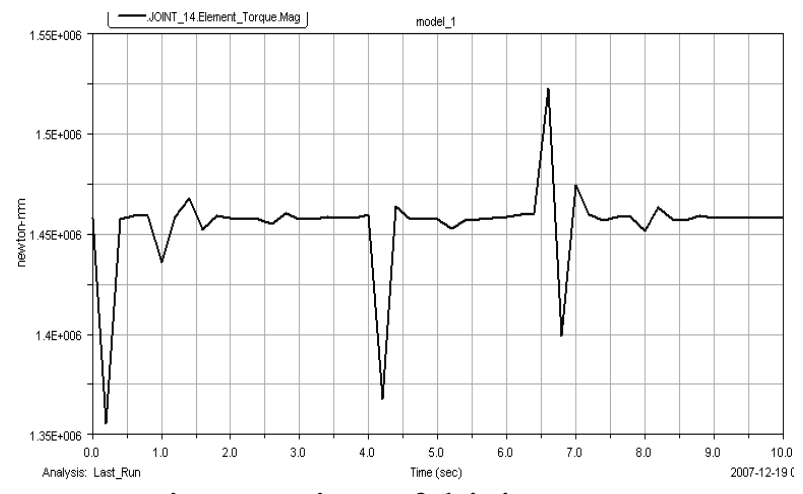

Fig. 5 Variety of driving torque

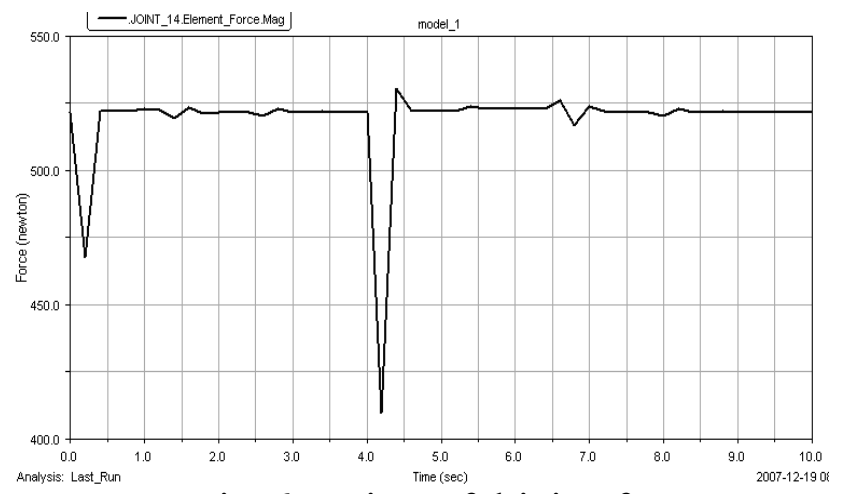

Fig. 6 Variety of driving force

Centriod's Trajectory in Invert Process. Model's centroid is at intersection of rolling shaft's center line with lifting lug's vertical certain line. This is the ideal centroid position, and its coordinate is $(-292.5136,-1440.82,553.3777)$. Passing by $10 \mathrm{~s}$ rolling, the ADAMS find out centriod's trajectory of X, Y, Z coordinate, and the variety curve was shown in the reference [4]. The coordinates of centriods each $0.2 \mathrm{~s}$ were calculated by ADAMS software, and then calculate two points' distance. The distance at $6.6 \mathrm{~s}$ is $268.9 \mathrm{~mm}$, so the maximum torque is also at this time.

\section{Conclusion}

The model of new upender clamp was built by use of SolidWorks and the reliability analysis and motion simulation was further performed in ADAMS. From the analysis results, lots of parameters were calculated. The reliability analysis results show that the clamp pressure was satisfied with the schedule design aim. The motion simulation results show driving force of upender process isn't very big. This illustrates that characteristics of the equipment came to energy saving purpose, and could also increase improve the upender efficiency. The results can be the key reference to select a suitable electric motor according to upender torque.

\section{References}

[1] Lu Fang, Design and application of electrical coil upender rig, Steel Rolling, 23(3) (2006)69-70. (in Chinese)

[2] Bretislav.Zuber, Coil handling device, NRC Research Press, Canada, 1982.

[3] Liao Chuanjun, Huang Weifeng, Suo Shuangfu, et al., Fluid-solid strong-interaction model of mechanical seals in reactor coolant pumps, Science China(Technological Sciences), 9(2011)89-92.

[4] F.H Wang, A.Y Jiao, L. L, et al., The performance research and dynamic analysis for new steel coil upender clamping device, Materials Science Forum, 628-629(2009) 73-76.

[5] Chen Liping, Zhang Yunqing, Ren Weiqun, et al., Dynamic analysis of mechanic and ADAMS-using course, TsingHua Universal, Beijing, 2005. (in Chinese) 